Solving $L_{1}$-CTA in 3D tables by an interior-point method for primal block-angular problems

$\begin{array}{cc}\text { Jordi Castro } & \text { Jordi Cuesta } \\ \begin{array}{c}\text { Dept. of Stat. and Operations Research } \\ \text { Universitat Politècnica de Catalunya }\end{array} & \begin{array}{c}\text { Dept. of Chemical Engineering } \\ \text { jordi. castro@upc.edu }\end{array} \\ \text { Universitat Rovira i Virgili } \\ \text { jordi.cuesta@urv. cat } \\ \text { March 2011 }\end{array}$

Report available from http://www-eio.upc.es/ jcastro 



\title{
Solving $L_{1}$-CTA in 3D tables by an interior-point method for primal block-angular problems
}

\author{
Jordi Castro · Jordi Cuesta
}

the date of receipt and acceptance should be inserted later

\begin{abstract}
The purpose of the field of statistical disclosure control is to avoid that no confidential information can be derived from statistical data released by, mainly, national statistical agencies. Controlled tabular adjustment (CTA) is an emerging technique for the protection of statistical tabular data. Given a table to be protected, CTA looks for the closest safe table. In this work we focus on CTA for three-dimensional tables using the $L_{1}$ norm for the distance between the original and protected tables. Three $L_{1}$-CTA models are presented, giving rise to six different primal block-angular structures of the constraint matrices. The resulting linear programming problems are solved by a specialized interior-point algorithm for this constraints structure, which solves the normal equations by a combination of Cholesky factorization and preconditioned conjugate gradients (PCG). In the past this algorithm shown to be one of the most efficient approaches for some classes of block-angular problems. The effect of quadratic regularizations is also analyzed, showing that for three of the six primal block-angular structures the performance of PCG is guaranteed to improve. Computational results are reported for a set of large instances, which provide linear optimization problems of up to 50 millions of variables and 25 millions of constraints. The specialized interior-point algorithm is compared with the state-of-the-art barrier solver of the CPLEX 12.1 package, showing to be a more efficient choice for very large $L_{1}$-CTA instances.
\end{abstract}

Keywords interior-point methods · primal block-angular problems · preconditioned conjugate gradient $\cdot$ regularizations $\cdot$ large-scale computational optimization · statistical tabular data protection · controlled tabular adjustment

Mathematics Subject Classification (2000) 90C90 · 90C06 · 90C08 · 90C51

Jordi Castro $(\bowtie)$

Dept. of Statistics and Operations Research, Universitat Politècnica de Catalunya, Jordi Girona 1-3, 08034 Barcelona, Catalonia, Spain. Tel.: +34-93-4015854. Fax.: +34-93-4015855. E-mail: jordi.castro@upc.edu, http://www-eio.upc.es/ jcastro

Jordi Cuesta

Statistics and Operations Research unit, Dept. of Chemical Engineering, Universitat Rovira i Virgili, Avda. Països Catalans 26, 43007 Tarragona, Catalonia, Spain. E-mail: jordi.cuesta@urv.cat 


\section{Introduction}

Minimum-distance controlled tabular adjustment, or CTA for short, (Dandekar and Cox, 2002; Castro, 2006) is an emerging technique for the protection of statistical tabular data (Hundepool et al, 2010). This is a major concern for national statistical institutes, which must guarantee that individual information cannot be disclosed from released data. Tabular data is obtained by crossing two or more variables in a file of microdata, e.g., city, age, and profession. The Cartesian product of values for these variables provides a set of cells. For each cell, the table reports either the number of individuals (frequency or contingency tables) or information about another variable, e.g., salary (magnitude tables). Some of the state-of-the-art research in this field can be found in the recent monographs Domingo-Ferrer and Saigin (2008); Domingo-Ferrer and Magkos (2010).

Given a table to be protected, CTA looks for the closest safe table, $L_{1}$ and $L_{2}$ being the two more practical distances. We will refer as $L_{1}$-CTA and $L_{2}$-CTA to these two particular variants. CTA is formulated as a mixed integer linear or quadratic optimization problem, with binary variables. It is a difficult combinatorial optimization problem even for medium size tables, and some heuristic approaches have been recently considered for $L_{1}$-CTA (González and Castro, 2011). If the binary variables are a priori fixed, the resulting linear or quadratic optimization problems are still challenging for very large instances. In practice, interior-point methods have shown to be more efficient than simplex algorithms for linear $L_{1}$-CTA instances (Castro, 2006). As it will be outlined in the paper, the constraint matrix associated to threedimensional (3D) tables (i.e., crossing three categorical variables) exhibits a primal block-angular structure (Castro, 2007b). In particular, the resulting $L_{2}$-CTA formulation in $3 \mathrm{D}$ tables is a quadratic multicommodity flow problem, and the specialized interior-point algorithm of Castro (2000) can be used. This specialized interior-point algorithm solves the normal equations at each interior-point iteration by a sensible scheme that combines Cholesky factorizations for the diagonal separable block constraints, and a preconditioned conjugate gradient (PCG) for the linking constraints. This fact was exploited in Castro (2005, 2007a); Castro and Cuesta (2011) to solve very large $L_{2}$-CTA instances. For example, some instances of 10 millions variables and 210000 constraints were solved with the specialized algorithm in 51 seconds, while CPLEX 11 needed 35000 seconds (Castro and Cuesta, 2011).

In this work we extend this approach to linear $L_{1}$-CTA in $3 \mathrm{D}$ tables. Due to the absolute value in the objective function, the resulting formulations are no longer multicommodity flow problems. But they still exhibit a primal block-angular structure, which can be dealt with the specialized interior-point algorithm. Three different models for $L_{1}$-CTA will be provided, each of them accepting two different primal blockangular structures. This amounts to six different primal block-angular structures. They are implemented and solved with the specialized interior-point algorithm. As it will be shown, a quadratic regularization term is instrumental to (partially) reproduce the good behaviour of the specialized algorithm for $L_{2}$-CTA. A set of 17 instancesranging from 500 to 50 millions variables and 320 to 25 millions constraints-will be used for the computational results. It will be shown that one of the six primal block- 
angular structures considered is efficient enough to outperform the state-of-the-art interior-point solver of CPLEX 12.1 in the largest instances.

The structure of the document is as follows. Section 2 outlines the formulation of the CTA problem, presents three different linear programming formulations for $L_{1}$ CTA, and shows the primal block-angular structure of CTA for 3D tables. Section 3 overviews the specialized interior-point algorithm for primal block-angular problems, and presents the main results relating the quality of the preconditioner and the presence of quadratic terms in the barrier problem (including quadratic regularizations). Section 4 presents six different primal block-angular structures for the three linear programming formulations of $L_{1}$-CTA, two for each of them; they differ in the definition of the block and linking constraints. Finally, Section 5 reports computational results with an implementation of the six different primal block-angular structures, which are solved through the specialized interior-point algorithm.

\section{The $L_{1}$-CTA problem}

Any CTA instance can be represented by the following parameters:

- A set of cells $a_{i}, i \in \mathscr{N}$, that satisfy a set linear relations $M_{j} a=b_{j}, j \in \mathscr{M}$. In matrix form these relations can be written $M a=b, a \in \mathbb{R}^{|\mathscr{N}|}, M \in \mathbb{R}^{|\mathscr{M}| \times|\mathscr{N}|}$ and $b \in{ }^{|\mathscr{M}|}$ being, respectively, the vector of $a_{i}$ 's, the matrix of table constraints, and the right-hand-side for these constraints.

- A lower and upper bound for each cell $i \in \mathscr{N}$, respectively $\underline{a}_{i}$ and $\bar{a}_{i}$, which are considered to be known by any attacker. If no previous knowledge is assumed for cell $i \underline{a}_{i}=0$ ( $\underline{a}_{i}=-\infty$ if $a \geq 0$ is not required) and $\bar{a}_{i}=+\infty$ can be used. The vectors of $\underline{a}_{i}$ 's and $\bar{a}_{i}$ 's are denoted as $\underline{a}$ and $\bar{a}$.

- A set $\mathscr{S} \subseteq \mathscr{N}$ of indices of sensitive or confidential cells. This cells are a priori determined by some sensitivity rules. Discussing this sensitivity rules is out of the scope of this work. Information about these rules can be found in Hundepool et al (2010).

- A lower and upper protection level for each confidential cell $i \in \mathscr{S}$, respectively $l p l_{i}$ and $u p l_{i}$, such that the released values $y_{i}, i \in \mathscr{N}$, satisfy either $y_{i} \geq a_{i}+u p l_{i}$ or $y_{i} \leq a_{i}-l p l_{i}$. The vector of $y_{i}$ 's is denoted as $y$.

CTA aims at finding the closest safe values $y_{i}, i \in \mathscr{N}$, according to some distance $L$, that makes the released table safe. This involves the solution of the following optimization problem:

$$
\begin{array}{ll}
\min _{y} & \|y-a\|_{L} \\
\text { s. to } & M y=b \\
& \underline{a} \leq y \leq \bar{a} \\
& y_{i} \leq a_{i}-l p l_{i} \text { or } \quad y_{i} \geq a_{i}+u p l_{i} \quad i \in \mathscr{S} .
\end{array}
$$


Problem (1) can also be formulated in terms of deviations from the current cell values. Defining $x=y-a, \underline{x}=\underline{a}-a$, and $\bar{x}=\bar{a}-a$, (1) can be recast as:

$$
\begin{array}{ll}
\min _{x} & \|x\|_{L} \\
\text { s. to } & M x=0 \\
& x \leq x \leq \bar{x} \\
& x_{i} \leq-l p l_{i} \text { or } x_{i} \geq u p l_{i} \quad i \in \mathscr{S},
\end{array}
$$

$x \in \mathbb{R}^{|\mathscr{N}|}$ being the vector of deviations. Problem (2) is a combinatorial optimization problem due to the disjunctive constraints. However, if the protection senses are a priori fixed for sensitive cells (i.e., only one of the two constraints $x_{i} \leq-l p l_{i}$ or $x_{i} \geq u p l_{i}$ is kept and the other is removed, for $i \in \mathscr{S}$ ), then, using the $L_{1}$ norm, (2) can be written as the following problem

$$
\begin{array}{ll}
\min _{x} & \sum_{i \in \mathscr{N}}\left|x_{i}\right| \\
\text { s. to } & M x=0 \\
& l \leq x \leq u,
\end{array}
$$

where $l, u \in \mathbb{R}^{|\mathscr{N}|}$ are easily obtained from $\underline{x}, \bar{x}, l p l_{i}$ and $u p l_{i}$, once the protection sense has been fixed.

2.1 Three linear programming formulations for (3)

The specialized interior-point solver outlined in Section 3 considers zero lower bounds for all the variables. The next three formulations meet this requirement.

The first formulation considers the change of variable $z=x-l$, such that (3) is equivalent to

$$
\begin{array}{ll}
\min _{z} & \sum_{i \in \mathscr{N}}\left|z_{i}+l_{i}\right| \\
\text { s. to } & M z=-A l \\
& 0 \leq z \leq u-l .
\end{array}
$$

Adding extra variables $t \in \mathbb{R}^{|\mathscr{N}|}$ for modeling the absolute value, we finally obtain the first model:

$$
\begin{array}{ll}
\min _{z, t} & \sum_{i \in \mathscr{N}} t_{i} \\
\text { s. to } & M z=-A l \\
& t \geq z+l \\
& t \geq-(z+l) \\
& 0 \leq z \leq u-l, \quad t \geq 0 .
\end{array}
$$

The second model is directly obtained from (3) by considering the standard change of variable when dealing with $L_{1}$ norms $x=x^{+}-x^{-}$, such that $\left|x_{i}\right|=x_{i}^{+}+x_{i}^{-}$:

$$
\begin{array}{cl}
\min _{x^{+}, x^{-}} & \sum_{i \in \mathscr{N}}\left(x_{i}^{+}+x_{i}^{-}\right) \\
\text {s. to } & M\left(x^{+}-x^{-}\right)=0 \\
& x^{+}-x^{-} \leq u \\
& x^{+}-x^{-} \geq l \\
& x^{+} \geq 0, \quad x^{-} \geq 0 .
\end{array}
$$


A third model, with less constraints but more variables, is obtained from (4) by considering the change of variable $z=x^{+}-x^{-}-l$. The third formulation is

$$
\begin{aligned}
\min _{z, x^{+}, x^{-}} & \sum_{i \in \mathscr{N}}\left(x_{i}^{+}+x_{i}^{-}\right) \\
\text {s. to } & M z=-M l \\
& z=x^{+}-x^{-}-l \\
& 0 \leq z \leq u-l, \quad x^{+} \geq 0, \quad x^{-} \geq 0 .
\end{aligned}
$$

2.2 The structure of table constraints in 3D tables

Crossing three categorical variables of, respectively, $r+1, c+1$ and $\kappa+1$ categories, we obtain a 3D table (i.e., a cube of data). The last category of each variable corresponds to marginal values, which, in practice, want to be published and can not be perturbed. The linear relations $M x=0$ of a 3D table are

$$
\begin{array}{ll}
\sum_{i_{1}=1}^{r} x_{i_{1} i_{2} i_{3}}=0 & i_{2}=1 \ldots c, i_{3}=1 \ldots \kappa \\
\sum_{i_{2}=1}^{c} x_{i_{1} i_{2} i_{3}}=0 & i_{1}=1 \ldots r, i_{3}=1 \ldots \kappa \\
\sum_{i_{3}=1}^{\kappa} x_{i_{1} i_{2} i_{3}}=0 & i_{1}=1 \ldots r, i_{2}=1 \ldots c .
\end{array}
$$

Matrix $M$ can be written in a primal block-angular form by exploiting the structure

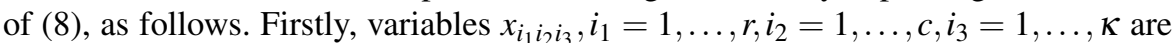
reordered according to $i_{3}$, i.e., $x=\left(x_{i_{1} i_{2} 1}^{T}, \ldots, x_{i_{1} i_{2} \kappa}^{T}\right)^{T} i_{1}=1, \ldots, r, i_{2}=1, \ldots, c$. Each group for a particular $i_{3}$ contains $r c$ variables, and it is associated to one slice of the 3D cube of data. Secondly, constraints (8a)-(8b) are set first, and ordered according to $i_{3}$. Each group for a particular $i_{3}$ contains $r+c$ constraints. The remaining $r c$ constraints $(8 \mathrm{c})$ are moved to end positions. The resulting constraint matrix structure is

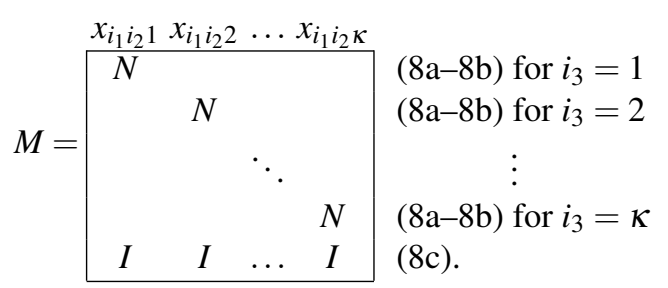

$N \in \mathbb{R}^{m \times n}$ denotes the structure of constraints ( $8 \mathrm{a}-8 \mathrm{~b}$ ), where $m=r+c-1$ and $n=r c . I \in \mathbb{R}^{n \times n}$ are identity matrices related to constraints (8c). In addition, it was shown that $N$ (related to the constraints and cells of a particular slice of the cube of data) is a node-arc incidence network matrix (Castro, 2007b) (one constraint is thus redundant, and this explains that the number of rows of $N$ is $r+c-1$ instead of $r+c$ ). Therefore, $M$ has a multicommodity network structure with equality linking constraints. 
It is worth noting that if the Euclidean distance $L_{2}$ is considered, the general formulation of $L_{1}$-CTA in $3 \mathrm{D}$ tables (3) should be replaced by

$$
\begin{array}{ll}
\min _{x} & \sum_{i \in \mathscr{N}} x_{i}^{2} \\
\text { s. to } & M x=0 \\
& l \leq x \leq u .
\end{array}
$$

Problem (10) is a quadratic multicommodity flow problem, and the specialized interiorpoint algorithm of Section 3 has shown to be the most efficient approach for this kind of problems (Castro, 2005; Castro and Cuesta, 2011). The three models for $L_{1}$-CTA (5)-(7) developed in Subsection 2.1 are no longer multicommodity problems since they involve additional constraints. In Section 4 we will show that they can be solved by the specialized interior-point algorithm.

\section{Overview of the specialized interior- point algorithm for primal block-angular problems}

The specialized interior-point algorithm was introduced for multicommodity flow problems in Castro (2000) and later extended to any primal block-angular structure in Castro (2007a). It considers the very general form of a primal block-angular problem

$$
\begin{aligned}
& \min \sum_{i=0}^{k}\left(c^{i^{T}} x^{i}+x^{i} Q_{i} x^{i}\right) \\
& \text { s. to }\left[\begin{array}{lllll}
N_{1} & & & \\
& N_{2} & & \\
& & \ddots & \\
& & & N_{k} \\
& & & & \\
L_{1} & L_{2} & \ldots & L_{k} & I
\end{array}\right]\left[\begin{array}{c}
x^{1} \\
x^{2} \\
\vdots \\
x^{k} \\
x^{0}
\end{array}\right]=\left[\begin{array}{c}
b^{1} \\
b^{2} \\
\vdots \\
b^{k} \\
b^{0}
\end{array}\right] \\
& 0 \leq x^{i} \leq u^{i} \quad i=0, \ldots, k \text {. }
\end{aligned}
$$

Matrices $N_{i} \in \mathbb{R}^{m_{i} \times n_{i}}$ and $L_{i} \in \mathbb{R}^{l \times n_{i}}, i=1, \ldots, k$, respectively define the block-diagonal and linking constraints, $k$ being the number of blocks. Vectors $x^{i} \in \mathbb{R}^{n_{i}}, i=1, \ldots, k$, are the variables for each block. $x^{0} \in \mathbb{R}^{l}$ are the slacks of the linking constraints. $b^{i} \in \mathbb{R}^{m_{i}}, i=1, \ldots, k$, is the right-hand-side vector for each block of constraints, whereas $b^{0} \in \mathbb{R}^{l}$ is for the linking constraints. The upper bounds for each group of variables are defined by $u^{i}, i=0, \ldots, k$. If needed, equality constraints may be defined with this formulation by imposing (close to) zero upper bounds on the slacks. $c^{i} \in \mathbb{R}^{n_{i}}$ and $Q_{i} \in \mathbb{R}^{n_{i} \times n_{i}}, i=1, \ldots, k$, define the linear and quadratic costs for each group of variables. We restrict our considerations to the separable case where $Q_{i}, i=0, \ldots, k$, are diagonal positive semidefinite matrices. Although the $L_{1}$-CTA formulations of this work are linear optimization problems, quadratic terms may be used for regularizing the algorithm (Castro and Cuesta, 2011). 
The specialized interior-point algorithm solves the normal equations at each interiorpoint iteration. The expression of normal equations (see, for instance, Wright (1996) for full details) is

$$
\left(A \Theta A^{T}\right) \Delta y=g
$$

where $\Theta$ is a positive definite diagonal matrix of blocks $\Theta_{i}, i=0, \ldots, k, \Delta y$ is the direction of dual variables, and $g$ is some right-hand-side $g$. Exploiting the structure of $A$ and $\Theta$ in (11) the matrix of (12) can be recast as

$$
\begin{aligned}
A \Theta A^{T}= & {\left[\begin{array}{ccc|c}
N_{1} \Theta_{1} N_{1}^{T} & & & N_{1} \Theta_{1} L_{1}^{T} \\
& \ddots & & \vdots \\
& & N_{k} \Theta_{k} N_{k}^{T} & N_{k} \Theta_{k} L_{k}^{T} \\
& & & \\
\hline & & & \\
L_{1} \Theta_{1} N_{1}^{T} & \ldots & L_{k} \Theta_{k} N_{k}^{T} & \Theta_{0}+\sum_{i=1}^{k} L_{i} \Theta_{i} L_{i}^{T}
\end{array}\right] } \\
= & {\left[\begin{array}{cc}
B & C \\
C^{T} & D
\end{array}\right], }
\end{aligned}
$$

$B \in \mathbb{R}^{\tilde{m} \times \tilde{m}}\left(\tilde{m}=\sum_{i=1}^{k} m_{i}\right), C \in \mathbb{R}^{\tilde{m} \times l}$ and $D \in \mathbb{R}^{l \times l}$ being the blocks of $A \Theta A^{T}$.

Appropriately partitioning $g$ and $\Delta y$ in (12), the normal equations can be written as

$$
\left[\begin{array}{cc}
B & C \\
C^{T} & D
\end{array}\right]\left[\begin{array}{l}
\Delta y_{1} \\
\Delta y_{2}
\end{array}\right]=\left[\begin{array}{l}
g_{1} \\
g_{2}
\end{array}\right]
$$

By eliminating $\Delta y_{1}$ from the first group of equations of (14), we obtain

$$
\begin{aligned}
\left(D-C^{T} B^{-1} C\right) \Delta y_{2} & =\left(g_{2}-C^{T} B^{-1} g_{1}\right) \\
B \Delta y_{1} & =\left(g_{1}-C \Delta y_{2}\right) .
\end{aligned}
$$

System (16) is solved by a Cholesky factorization for each diagonal block $N_{i} \Theta_{i} N_{i}^{T}, i=$ $1 \ldots k$, of $B$. The system with matrix $D-C^{T} B^{-1} C$, the Schur complement of (14), is solved by a PCG. The dimension of this system is $l$, which is the number of linking constraints. In Castro (2000) it was proved that, under some conditions, which are guaranteed in our setting, the inverse of $\left(D-C^{T} B^{-1} C\right)$ can be computed as

$$
\left(D-C^{T} B^{-1} C\right)^{-1}=\left(\sum_{i=0}^{\infty}\left(D^{-1}\left(C^{T} B^{-1} C\right)\right)^{i}\right) D^{-1} .
$$

The preconditioner $M^{-1}$, an approximation of $\left(D-C^{T} B^{-1} C\right)^{-1}$, is thus obtained by truncating the infinite power series (17) at some term $h$. The more the terms included, the better the preconditioner will be, at the expense of increasing the execution time of each PCG iteration. However, in general, $h=0$ or $h=1$ are reasonable choices, which in practice yield

$$
\begin{array}{ll}
M^{-1}=D^{-1} & \text { if } h=0, \\
M^{-1}=\left(I+D^{-1}\left(C^{T} B^{-1} C\right)\right) D^{-1} & \text { if } h=1 .
\end{array}
$$

This preconditioner, initially developed for multicommodity flows (Castro, 2000) can be applied to any primal block-angular problem (Castro, 2007a). 
3.1 Improving the effectiveness of the preconditioner

The effectiveness of the preconditioner depends on the spectral radius of matrix $D^{-1}\left(C^{T} B^{-1} C\right)$, which is always in $[0,1)($ Castro, 2000, Theorem 1$)$. The farther away from 1 is the spectral radius of $D^{-1}\left(C^{T} B^{-1} C\right)$, the better is the quality of the approximation of (17) obtained by truncation with $h=0$ or $h=1$. The next theorem and proposition from Castro and Cuesta (2011) show that a quadratic term in the objective function effectively reduces this spectral radius.

Theorem 1 Let $A$ be the constraint matrix of problem (11), with full row rank matrices $N_{i} \in \mathbb{R}^{m_{i} \times n_{i}} i=1, \ldots, k$, and at least one full row rank matrix $L_{i} \in \mathbb{R}^{l \times n_{i}}$, $i=1, \ldots, k$. Let $\Theta$ be the diagonal positive definite matrix of (12), and $B \in \mathbb{R}^{\tilde{m} \times \tilde{m}}$, $C \in \mathbb{R}^{\tilde{m} \times l}$ and $D \in \mathbb{R}^{l \times l}$ the submatrices of $A \Theta A^{T}$ defined in (13). Then, the spectral radius $\rho$ of $D^{-1}\left(C^{T} B^{-1} C\right)$ is bounded by

$$
0 \leq \rho \leq \max _{j \in\{1, \ldots, l\}} \frac{\gamma_{j}}{\left(\frac{u_{j}}{v_{j}}\right)^{2} \Theta_{0 j}+\gamma_{j}}<1,
$$

where $u$ is the eigenvector (or one of the eigenvectors) of $D^{-1}\left(C^{T} B^{-1} C\right)$ for $\rho ; \gamma_{j}, j=$ $1, \ldots, l$, and $V=\left[V_{1} \ldots V_{l}\right]$, are respectively the eigenvalues and matrix of columnwise eigenvectors of $\sum_{i=1}^{k} L_{i} \Theta_{i} L_{i}{ }^{T} ; v=V^{T} u$; and, abusing of notation, we assume that for $v_{j}=0,\left(u_{j} / v_{j}\right)^{2}=+\infty$.

Proposition 1 Let assume the hypotheses of Theorem 1, and consider a linear problem and a quadratic one obtained by adding (likely small) quadratic costs $Q_{i} \succ 0$, $i=1, \ldots, k$. Assume $\hat{u}_{j} / \hat{v}_{j} \leq u_{j} / v_{j}, j=1, \ldots, l$, where "hatted" and "non-hatted" terms refer, respectively, to the linear and quadratic problems, and $u$ and $v$ are defined as in Theorem 1. Then bound (18) is smaller for the quadratic than for the linear problem.

The technical assumption $\hat{u}_{j} / \hat{v}_{j} \leq u_{j} / v_{j}, j=1, \ldots, l$ in Proposition 1 is needed to guarantee that the bound (18) is smaller if a quadratic term is added to a linear problem. The fulfillment of this assumption is problem dependent, and, for a general problem, it may not be easy to check. However, as it will be shown in Section 5 , matrices $L_{i}, i=1, \ldots, k$, of some formulations of $L_{1}$-CTA satisfy this requirement. Therefore, it makes sense to consider a quadratic regularization term in the objective function to reduce the spectral radius, i.e., to improve the quality of the preconditioner. In this work we will consider a quadratic regularization in the barrier function of the interior-point algorithm. The particular form of this regularization term is

$$
R(x)=\mu \frac{1}{2} x^{T} Q x \quad Q=t \delta / \mu_{0} I,
$$

where $x$ is the vector of variables, $\mu \in \mathbb{R}$ is the parameter of the barrier function, $t \in \mathbb{Z}$ is the number of interior-point iteration, $\mu_{0} \in \mathbb{R}$ the value of the barrier parameter at the first interior-point iteration, and $\delta \in \mathbb{R}$ a parameter to be provided by the user for initializing the regularization matrix $Q$ at the first iteration. Note that at the first iteration the value of the regularization is $R(x)=\mu_{0} x^{T}\left(1 \cdot \delta / \mu_{0} I\right) x=\delta x^{T} x$, and as we approach the optimal solution $\mu \rightarrow 0$, thus, $R(x) \rightarrow 0$. Full details about this regularized version of the algorithm can be found in Castro and Cuesta (2011). 


\section{Primal block-angular structures for $L_{1}$-CTA in 3D tables}

Let us consider that the vectors of variables $z, t, x^{+}, x^{-} \in \mathbb{R}^{\kappa n}$ and bounds $l, u \in \mathbb{R}^{\kappa n}$, which intervene in the definition of models (5)-(7), are partitioned in $\kappa$ blocks, one for each category of the third dimension, i.e., $z=\left(\left(z^{1}\right)^{T}, \ldots,\left(z^{\kappa}\right)^{T}\right)^{T}$, and similarly for the other vectors. Each block $z^{i}, t^{i},\left(x^{+}\right)^{i},\left(x^{-}\right)^{i} \in \mathbb{R}^{n}, i=1, \ldots, \kappa$ is related to the variables and bounds for a particular slice of the $3 \mathrm{D}$ cube of data, which contains $n=r c$ cells and $m=r+c-1$ table constraints.

Exploiting the above partitioning of vectors and the structure of $M$ in (9), (5) is equivalent to

$$
\begin{array}{lll}
\min _{z, t} & \sum_{i=1}^{\kappa} e^{T} t^{i} & \\
\text { s. to } & N z^{i}=-N l^{i} & \\
& & \\
& \sum_{i=1}^{\kappa} z^{i}=-\sum_{i=1}^{\kappa} l^{i} & \\
& z_{i}-t^{i} \leq-l^{i} & \\
& -z_{i}-t^{i} \leq l^{i} & i=1, \ldots, \kappa \\
& 0 \leq z^{i} \leq u^{i}-l^{i}, \quad t^{i} \geq 0 & i=1, \ldots, \kappa \\
&
\end{array}
$$

where $e \in \mathbb{R}^{n}$ is a vector of 1's. The constraints matrix $A$ defined by (20b)-(20e) accept different structures, depending on the reordering of variables and constraints considered. We will consider the following two:
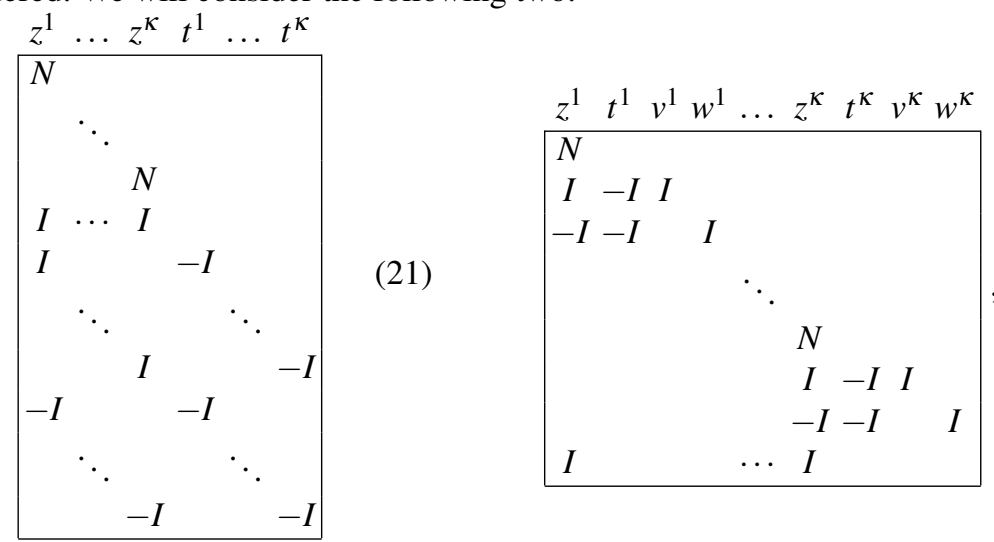

where $v^{i}, w^{i}, i=1, \ldots, \kappa$ are, respectively, the slacks of (20d) and (20e), and $I \in \mathbb{R}^{n \times n}$ are identity matrices. The structure of (21) is obtained with the natural ordering of (20b)-(20e). It matches the primal block-angular structure of (11) by setting $k=2 \kappa$, $N_{i}=N, m_{i}=m, n_{i}=n$ for $i=1, \ldots, \kappa, N_{i} \in \mathbb{R}^{0 \times n}$ for $i=\kappa+1, \ldots, 2 \kappa$ (empty matrix); $L_{i} \in \mathbb{R}^{l \times n}, i=1, \ldots, 2 \kappa$ are defined by the blocks of (21) with matrices $I$ and $-I$ for each $z^{i}$ and $t^{i}$, where $l=(2 \kappa+1) n$. In this scheme the block constraints are small, while the number of linking constraints is very large. The structure of (22) is obtained after reordering the variables and constraints, and transforming (20d) and 
(20e) to equalities. This structure also matches (11), where $k=\kappa$,

$$
N_{i}=\left[\begin{array}{ccc}
N & & \\
I & -I & I \\
-I-I & I
\end{array}\right], \quad L_{i}=\left[\begin{array}{llll}
I & 0 & 0 & 0
\end{array}\right], \quad i=1, \ldots, k
$$

$0 \in \mathbb{R}^{n \times n}$ is a matrix of 0 's, $m_{i}=m+2 n, n_{i}=4 n$, for $i=1, \ldots, k$, and $l=n$.

Similarly, exploiting the structure of $M,(6)$ is equivalent to

$$
\begin{array}{lll}
\min _{x^{+}, x^{-}} & \sum_{i=1}^{\kappa} e^{T}\left(x^{+i}+x^{-i}\right) & \\
\text { s. to } & N\left(x^{+i}-x^{-i}\right)=0 & i=1, \ldots, \kappa \\
& \sum_{i=1}^{\kappa}\left(x^{+i}-x^{-i}\right)=0 & \\
& x^{+i}-x^{-i} \leq u^{i} & i=1, \ldots, \kappa \\
& -x^{+i}+x^{-i} \leq-l^{i} & i=1, \ldots, \kappa \\
& x^{+i} \geq 0, \quad x^{-i} \geq 0 & i=1, \ldots, \kappa .
\end{array}
$$

The two particular structures, with and without reordering, of the constraints matrix $A$ defined by (24b)-(24e) are

$$
\begin{array}{|cccccc}
x^{+1} & x^{-1} & \ldots & x^{+\kappa} & x^{-\kappa} \\
\hline N & -N & & & \\
& & \ddots & & \\
& & & N & -N \\
I & -I & \ldots & I & -I \\
I & -I & & & \\
& & \ldots & & \\
& & & I & -I \\
-I & I & & & \\
& & \cdots & & \\
& & & -I & I \\
\hline
\end{array}
$$$$
\begin{array}{|ccccccccc}
x^{+1} & x^{-1} & v^{1} & w^{1} & \ldots & x^{+\kappa} & x^{-\kappa} & v^{\kappa} & w^{\kappa} \\
\hline N & -N & & & & & & & \\
I & -I & I & & & & & & \\
-I & I & & I & & & & & \\
& & & & \ddots & & & & \\
& & & & & N & -N & \\
& & & & & I & -I & I & \\
& & & & & & \\
& & & & & -I & I & & I \\
I & -I & & & \cdots & I & -I & \\
\hline
\end{array}
$$

where $v^{i}, w^{i}, i=1, \ldots, \kappa$ are, respectively, the slacks of (24d) and (24e). Both (25) and (26) match (11). For (25) we have $k=\kappa, N_{i}=[N-N], m_{i}=m, n_{i}=2 n$, $l=(2 \kappa+1) n$, and $L_{i} \in \mathbb{R}^{l \times 2 n}, i=1, \ldots, \kappa$ are defined by the blocks of (25) with matrices $I$ and $-I$ for $\left(x^{+i}, x^{-i}\right)$. On the other hand, for (26), $k=\kappa, m_{i}=m+2 n$, $n_{i}=4 n, l=n$, and

$$
N_{i}=\left[\begin{array}{ccc}
N & -N & \\
I & -I & I \\
-I & I & I
\end{array}\right], \quad L_{i}=\left[\begin{array}{llll}
I & -I & 0 & 0
\end{array}\right], \quad i=1, \ldots, k
$$


Finally, (7) is equivalent to

$$
\begin{array}{lll}
\min _{z, x^{+}, x^{-}} & \sum_{i=1}^{\kappa} e^{T}\left(x^{+i}+x^{-i}\right) & \\
\text { s. to } & N z^{i}=-N l^{i} & \\
& & \\
& \sum_{i=1}^{\kappa} z^{i}=-\sum_{i=1}^{\kappa} l^{i} & \\
& z^{i}-x^{+i}+x^{-i}=-l^{i} & \\
& 0 \leq z^{i} \leq u^{i}-l^{i}, \quad x^{+i} \geq 0, \quad x^{-i} \geq 0 & i=1, \ldots, \kappa
\end{array}
$$

The two particular structures, with and without reordering, of the constraints matrix $A$ defined by (28b)-(28d) are
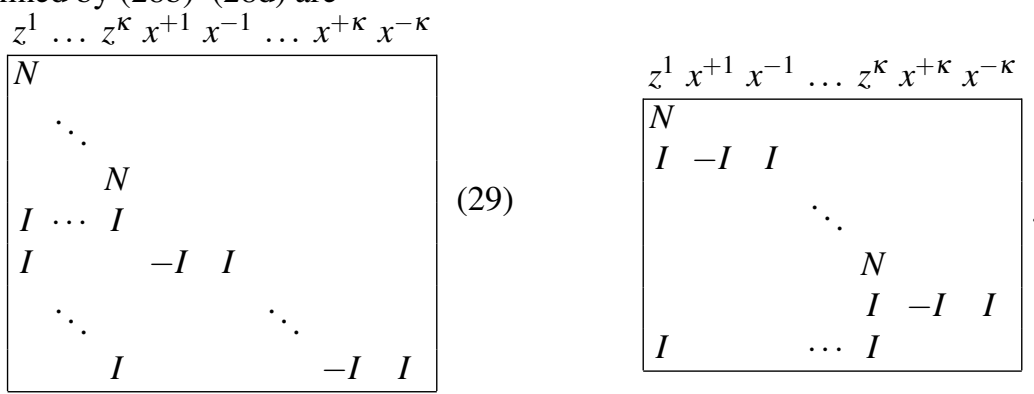

As for previous models, (29) and (30) match (11). For structure (29) $k=2 \kappa, N_{i}=$ $N, m_{i}=m, n_{i}=n$ for $i=1, \ldots, \kappa, N_{i} \in \mathbb{R}^{0 \times 2 n}$ for $i=\kappa+1, \ldots, 2 \kappa$ (empty matrix), $l=(\kappa+1) n, L_{i} \in \mathbb{R}^{l \times n}$ for $i=1, \ldots, \kappa$, and $L_{i} \in \mathbb{R}^{l \times 2 n}$ for $i=\kappa+1, \ldots, 2 \kappa$. For (30), $k=\kappa, m_{i}=m+n, n_{i}=3 n, l=n$, and

$$
N_{i}=\left[\begin{array}{ccc}
N & \\
I & -I & I
\end{array}\right], \quad L_{i}=\left[\begin{array}{lll}
I & 0 & 0
\end{array}\right], \quad i=1, \ldots, k, .
$$

The three pairs of structures (21)-(22), (25)-(26) and (29)-(30), amount to six different formulations of $L_{1}$-CTA which can be solved with the specialized algorithm of Section 3. Formulations (21), (25), (29) have in common very small block diagonal matrices $N_{i}$, but a large number $l$ of linking constraints. On the other hand, structures (22), (26) and (30) have larger diagonal matrices $N_{i}$ but significantly fewer linking constraints. A priori the latter seem to be more promising approaches, since the fewer linking constraints, the higher the performance of the specialized algorithm. The computational comparison made at the beginning of Section 5 between the two groups of formulations confirms this guess. Another advantage of formulations (22), (26) and (30) is that the topology of $N_{i}$ and $L_{i}$ is the same for all the blocks $i=1, \ldots, k$, which is a slight computational benefit when performing the symbolic factorizations in the interior-point algorithm. However, the most instrumental feature is that, as shown by below Proposition 2, the structures of matrices $L_{i}$ for (22), (26) and (30) satisfy the technical assumption $\hat{u}_{j} / \hat{v}_{j} \leq u_{j} / v_{j}, j=1, \ldots, l$, of Proposition 1. This guarantees that quadratic terms in the objective function (which can be added by a quadratic regularization (Castro and Cuesta, 2011)) will improve the performance of the algorithm. 
Proposition 2 Given the hypotheses of Theorem 1, let us consider the definition of $u$ and $v$ in that Theorem. Let $u, v$ be the vectors $\hat{u}$ and $\hat{v}$ for a quadratic problem obtained by adding a quadratic term to the linear objective. Then, for the constraint matrices A defined by (22), (26) and (30), it holds that $\hat{u}_{j} / \hat{v}_{j}=u_{j} / v_{j}, j=1, \ldots, l$.

Proof By definition, $u, v \in \mathbb{R}^{l}, v=V^{T} u$, where $V=\left[V_{1} \ldots V_{l}\right] \in \mathbb{R}^{l \times l}$, is the matrix of columnwise eigenvectors $V_{j}, j=1, \ldots, l$ of $\sum_{i=1}^{k} L_{i} \Theta_{i} L_{i}{ }^{T}$. Matrices $L_{i}, i=$ $1, \ldots, k$ for (22), (26) and (30) are, respectively, defined by (23), (27), (31). The matrices $\sum_{i=1}^{k} L_{i} \Theta_{i} L_{i}^{T}$ derived from (23), (27), (31) are thus, respectively, $\sum_{i=1}^{k} \Theta_{z^{i}}$, $\sum_{i=1}^{k}\left(\Theta_{x^{+i}}-\Theta_{x^{-i}}\right)$ and $\sum_{i=1}^{k} \Theta_{z^{i}}$, which are positive definite and diagonal. Therefore $V=I, v=I u=u$, and similarly for the linear objective $\hat{V}=I, \hat{v}=I \hat{u}=\hat{u}$, thus satisfying $\hat{u}_{j} / \hat{v}_{j}=u_{j} / v_{j}=1, j=1, \ldots, l$.

By Propositions 2 and 1 the bound of the spectral radius (18) is reduced if a quadratic regularization is added to the objective function. A it will be shown in Section 5, the regularized version of the specialized algorithm is by far more efficient than the non regularized version for large $L_{1}$-CTA instances, making it competitive against state-of-the-art interior-point implementations like CPLEX 12.1.

\section{Computational results}

The six different formulations (21)-(22), (25)-(26) and (29)-(30) have been implemented and solved with the specialized interior-point algorithm. We used a MATLAB implementation of the algorithm described in Castro (2007a), named PRBLOCK_IP. PRBLOCK_IP implements a standard infeasible path-following algorithm (Wright, 1996), which solves normal equations either through a Cholesky factorization, or through the specialized procedure. Note that neither the Mehrotra predictor-corrector (Mehrotra, 1992) nor the multiple centrality corrections (Gondzio, 1996) heuristics for higher order directions are not used, since, as shown in Castro (2000), they are not useful when PCG is applied in interior-point methods. For reasons of efficiency, Cholesky factorizations are performed through external precompiled routines. Specifically, the code uses the Ng-Peyton sparse Cholesky package Ng and Peyton (1993), hooked to MATLAB for the LIPSOL package Zhang (1998). The code also includes the regularization strategy described in Castro and Cuesta (2011). PRBLOCK_IP can be obtained for research purposes from http://www-eio.upc.es/ jcastro/prblock_ip.html.

Results with the barrier algorithm of CPLEX 12.1 are also reported. The CPLEX simplex solvers were not used, since interior-point algorithms have shown to be the most efficient option for the linear programming formulations of CTA (Castro, 2006).

MATLAB and CPLEX were hooked by a free software, available from http://www-eio. upc. es/ jcastro/software.html. It is worth noting that CPLEX 12.1 is a state-of-the-art code with highly optimized routines, while the Ng-Peyton package used by PRBLOCK_IP is an academic free implementation for sparse Cholesky factorizations. Therefore, the comparison between PRBLOCK_IP and CPLEX 12.1 is biased due to the quality of the implementation. All runs were carried out on a Dell PowerEdge 6950 server with four dual core AMD Opteron 8222 3.0 GHZ processors and 64 GB of RAM, without exploitation of parallelism capabilities. 
Table 1 Dimensions of $L_{1}$-CTA instances for formulations (21)-(22)

\begin{tabular}{|c|c|c|c|c|c|c|c|c|}
\hline \multirow[b]{2}{*}{ Instance } & \multicolumn{3}{|c|}{ Formulation (21) } & \multicolumn{3}{|c|}{ Formulation (22) } & \multirow[b]{2}{*}{ vars. } & \multirow[b]{2}{*}{ const. } \\
\hline & $k$ & $m_{i}^{(*)}$ & $n_{i}$ & $k$ & $m_{i}$ & $n_{i}$ & & \\
\hline CTA-5-5-5 & 10 & 9 & 25 & 5 & 59 & 100 & 500 & 320 \\
\hline CTA-10-10-5 & 10 & 19 & 100 & 5 & 219 & 400 & 2000 & 1195 \\
\hline CTA-15-15-10 & 20 & 29 & 225 & 10 & 479 & 900 & 9000 & 5015 \\
\hline CTA-20-20-20 & 40 & 39 & 400 & 20 & 839 & 1600 & 32000 & 17180 \\
\hline CTA-25-25-10 & 20 & 49 & 625 & 10 & 1299 & 2500 & 25000 & 13615 \\
\hline CTA-25-25-25 & 50 & 49 & 625 & 25 & 1299 & 2500 & 62500 & 33100 \\
\hline CTA-30-30-10 & 20 & 59 & 900 & 10 & 1859 & 3600 & 36000 & 19490 \\
\hline CTA-30-30-30 & 60 & 59 & 900 & 30 & 1859 & 3600 & 108000 & 56670 \\
\hline CTA-40-40-20 & 40 & 79 & 1600 & 20 & 3279 & 6400 & 128000 & 67180 \\
\hline CTA-50-50-10 & 20 & 99 & 2500 & 10 & 5099 & 10000 & 100000 & 53490 \\
\hline CTA-50-50-25 & 50 & 99 & 2500 & 25 & 5099 & 10000 & 250000 & 129975 \\
\hline CTA-50-50-50 & 100 & 99 & 2500 & 50 & 5099 & 10000 & 500000 & 257450 \\
\hline CTA-100-100-10 & 20 & 199 & 10000 & 10 & 20199 & 40000 & 400000 & 211990 \\
\hline CTA-100-100-25 & 50 & 199 & 10000 & 25 & 20199 & 40000 & 1000000 & 514975 \\
\hline CTA-200-200-50 & 100 & 399 & 40000 & 50 & 80399 & 160000 & 8000000 & 4059950 \\
\hline CTA-500-500-50 & 100 & 999 & 250000 & 50 & 500999 & 1000000 & 50000000 & 25299950 \\
\hline CTA-1000-500-20 & 40 & 1499 & 500000 & 20 & 1001499 & 2000000 & 40000000 & 20529980 \\
\hline
\end{tabular}

(*) Only for first $\kappa$ nonempty blocks

As MATLAB is an interpreted language, the overall execution time is meaningless. Following Castro (2007a), we only report the execution time spent in the external precompiled $\mathrm{Ng}$-Peyton Cholesky routines, including minimum degree ordering, symbolic factorization, numerical factorization, and numerical solution. Note that communication between MATLAB and the external precompiled Ng-Peyton Cholesky routines is made by value (copy of parameters). This means a significant overhead time for large instances, which is included in the time reported. For large instances, this reported time should be comparable to the total time spent by an efficient $\mathrm{C} / \mathrm{C}++$ implementation. For the runs with CPLEX 12.1 we provide the overall execution time. An efficient $\mathrm{C} / \mathrm{C}++$ version of PRBLOCK_IP is work in progress.

We generated a set of 17 3D CTA instances with a random generator of synthetic tables. The generator can be retrieved from http://www-eio.upc.es/ jcastro/CTA_3Dtables.html. Tables 1-3 report the dimensions of each instance, for, respectively, the formulations (21)-(22), (25)-(26) and (29)-(30). Instances are denoted as CTA- $c-r$ - $\kappa$, where $r, c$ and $\kappa$ are the number of categories of the variables of the $3 \mathrm{D}$ table. For each instance and formulation the tables report the number of blocks $(k)$, the number of constraints and variables for each block $\left(m_{i}\right.$ and $\left.n_{i}\right)$, and the overall number of constraints and variables of the linear problem ("vars." and "const."), including the slacks of inequality linking constraints. For formulations (21) and (29) columns $m_{i}$ provide the number of constraints of the first $\kappa$ nonempty diagonal blocks.

From tables 1-3 it is clear that, although the overall number of variables and constraints of the resulting linear problem is the same for the two formulations of each table, the dimension of the diagonal matrices $\left(m_{i} \times n_{i}\right)$ of formulations $(21),(25)$ and (29) is much smaller (or, equivalently, the number of linking constraints of formulations (21), (25) and (29) is much larger). Since the dimension of systems to be solved 
Table 2 Dimensions of $L_{1}$-CTA instances for formulations (25)-(26)

\begin{tabular}{|c|c|c|c|c|c|c|c|c|}
\hline \multirow[b]{2}{*}{ Instance } & \multicolumn{3}{|c|}{ Formulation (25) } & \multicolumn{3}{|c|}{ Formulation (26) } & \multirow[b]{2}{*}{ vars. } & \multirow[b]{2}{*}{ const. } \\
\hline & $k$ & $m_{i}$ & $n_{i}$ & $k$ & $m_{i}$ & $n_{i}$ & & \\
\hline CTA-5-5-5 & 5 & 9 & 50 & 5 & 59 & 100 & 500 & 320 \\
\hline CTA-10-10-5 & 5 & 19 & 200 & 5 & 219 & 400 & 2000 & 1195 \\
\hline CTA-15-15-10 & 10 & 29 & 450 & 10 & 479 & 900 & 9000 & 5015 \\
\hline CTA-20-20-20 & 20 & 39 & 800 & 20 & 839 & 1600 & 32000 & 17180 \\
\hline CTA-25-25-10 & 10 & 49 & 1250 & 10 & 1299 & 2500 & 25000 & 13615 \\
\hline CTA-25-25-25 & 25 & 49 & 1250 & 25 & 1299 & 2500 & 62500 & 33100 \\
\hline CTA-30-30-10 & 10 & 59 & 1800 & 10 & 1859 & 3600 & 36000 & 19490 \\
\hline CTA-30-30-30 & 30 & 59 & 1800 & 30 & 1859 & 3600 & 108000 & 56670 \\
\hline CTA-40-40-20 & 20 & 79 & 3200 & 20 & 3279 & 6400 & 128000 & 67180 \\
\hline CTA-50-50-10 & 10 & 99 & 5000 & 10 & 5099 & 10000 & 100000 & 53490 \\
\hline CTA-50-50-25 & 25 & 99 & 5000 & 25 & 5099 & 10000 & 250000 & 129975 \\
\hline CTA-50-50-50 & 50 & 99 & 5000 & 50 & 5099 & 10000 & 500000 & 257450 \\
\hline CTA-100-100-10 & 10 & 199 & 20000 & 10 & 20199 & 40000 & 400000 & 211990 \\
\hline CTA-100-100-25 & 25 & 199 & 20000 & 25 & 20199 & 40000 & 1000000 & 514975 \\
\hline CTA-200-200-50 & 50 & 399 & 80000 & 50 & 80399 & 160000 & 8000000 & 4059950 \\
\hline CTA-500-500-50 & 50 & 999 & 500000 & 50 & 500999 & 1000000 & 50000000 & 25299950 \\
\hline CTA-1000-500-20 & 20 & 1499 & 1000000 & 20 & 1001499 & 2000000 & 40000000 & 20529980 \\
\hline
\end{tabular}

Table 3 Dimensions of $L_{1}$-CTA instances for formulations (29)-(30)

\begin{tabular}{|c|c|c|c|c|c|c|c|c|}
\hline \multirow[b]{2}{*}{ Instance } & \multicolumn{3}{|c|}{ Formulation (29) } & \multicolumn{3}{|c|}{ Formulation (30) } & \multirow[b]{2}{*}{ vars. } & \multirow[b]{2}{*}{ const. } \\
\hline & $k$ & $m_{i}^{(*)}$ & $n_{i}$ & $k$ & $m_{i}$ & $n_{i}$ & & \\
\hline CTA-5-5-5 & 10 & 9 & 25 & 5 & 34 & 75 & 375 & 195 \\
\hline CTA-10-10-5 & 10 & 19 & 100 & 5 & 119 & 300 & 1500 & 695 \\
\hline CTA-15-15-10 & 20 & 29 & 225 & 10 & 254 & 675 & 6750 & 2765 \\
\hline CTA-20-20-20 & 40 & 39 & 400 & 20 & 439 & 1200 & 24000 & 9180 \\
\hline CTA-25-25-10 & 20 & 49 & 625 & 10 & 674 & 1875 & 18750 & 7365 \\
\hline CTA-25-25-25 & 50 & 49 & 625 & 25 & 674 & 1875 & 46875 & 17475 \\
\hline CTA-30-30-10 & 20 & 59 & 900 & 10 & 959 & 2700 & 27000 & 10490 \\
\hline CTA-30-30-30 & 60 & 59 & 900 & 30 & 959 & 2700 & 81000 & 29670 \\
\hline CTA-40-40-20 & 40 & 79 & 1600 & 20 & 1679 & 4800 & 96000 & 35180 \\
\hline CTA-50-50-10 & 20 & 99 & 2500 & 10 & 2599 & 7500 & 75000 & 28490 \\
\hline CTA-50-50-25 & 50 & 99 & 2500 & 25 & 2599 & 7500 & 187500 & 67475 \\
\hline CTA-50-50-50 & 100 & 99 & 2500 & 50 & 2599 & 7500 & 375000 & 132450 \\
\hline CTA-100-100-10 & 20 & 199 & 10000 & 10 & 10199 & 30000 & 300000 & 111990 \\
\hline CTA-100-100-25 & 50 & 199 & 10000 & 25 & 10199 & 30000 & 750000 & 264975 \\
\hline CTA-200-200-50 & 100 & 399 & 40000 & 50 & 40399 & 120000 & 6000000 & 2059950 \\
\hline CTA-500-500-50 & 100 & 999 & 250000 & 50 & 250999 & 750000 & 37500000 & 12799950 \\
\hline CTA-1000-500-20 & 40 & 1499 & 500000 & 20 & 501499 & 1500000 & 30000000 & 10529980 \\
\hline
\end{tabular}

${ }^{(*)}$ Only for first $\kappa$ nonempty blocks

by PCG in the the specialized interior-point algorithm is the number of linking constraints, (21), (25) and (29) seem not to be good candidates. This is confirmed by the results of Tables $4-6$. They provide the computational results obtained with, respectively, (21)-(22), (25)-(26) and (29)-(30), for a subset of small instances. Columns "It.", "PCG" and "CPU" provide the number of interior-point iterations, overall number of PCG iteration and CPU time. The maximum allowed number of interior-point 
Table 4 Results for a subset of small instances comparing (21) and (22)

\begin{tabular}{lrrrrrrr} 
& \multicolumn{3}{c}{ Formulation $(21)$} & & \multicolumn{3}{c}{ Formulation $(22)$} \\
\cline { 2 - 4 } \cline { 6 - 8 } Instance & It. & PCG & CPU & & It. & PCG & CPU \\
\hline CTA-5-5-5 & $51(9)$ & 4670 & 1.38 & & 200 & 280 & 0.34 \\
CTA-10-10-5 & $48(13)$ & 14851 & 4.93 & & 200 & 1724 & 0.77 \\
CTA-15-15-10 & $100(44)$ & 145745 & 152.09 & & $111(23)$ & 5382 & 4.57 \\
CTA-20-20-20 & $100(21)$ & 503361 & 1575.34 & & $200(118)$ & 6807 & 40.24 \\
CTA-25-25-10 & $98(36)$ & 411112 & 854.00 & & $93(6)$ & 17958 & 27.40 \\
CTA-25-25-25 & $100(20)$ & 1105761 & 6939.38 & & 200 & 5936 & 30.80 \\
CTA-30-30-10 & $117(40)$ & 672017 & 2475.10 & & $200(74)$ & 36583 & 77.22 \\
CTA-30-30-30 & $100(4)$ & 1458906 & 15883.20 & & $200(66)$ & 27319 & 343.88 \\
\hline
\end{tabular}

Table 5 Results for a subset of small instances comparing (25) and (26)

\begin{tabular}{lrrrrrrrr} 
& \multicolumn{3}{c}{ Formulation $(25)$} & & \multicolumn{3}{c}{ Formulation $(26)$} \\
\cline { 2 - 4 } \cline { 7 - 8 } Instance & It. & PCG & CPU & & It. & PCG & CPU \\
\hline CTA-5-5-5 & 18 & 65 & 0.06 & & 27 & 76 & 0.07 \\
CTA-10-10-5 & 48 & 1671 & 0.38 & & 58 & 502 & 0.22 \\
CTA-15-15-10 & 46 & 3247 & 2.92 & & 48 & 1951 & 1.47 \\
CTA-20-20-20 & 51 & 13722 & 30.22 & & $57(15)$ & 3528 & 11.40 \\
CTA-25-25-10 & 64 & 22556 & 37.33 & & 48 & 4594 & 6.79 \\
CTA-25-25-25 & 48 & 14437 & 95.68 & & 36 & 1454 & 6.17 \\
CTA-30-30-10 & 54 & 6501 & 15.89 & & $75(21)$ & 7806 & 20.30 \\
CTA-30-30-30 & 58 & 13106 & 161.67 & & $70(20)$ & 5624 & 90.06 \\
\hline
\end{tabular}

Table 6 Results for a subset of small instances comparing (29) and (30)

\begin{tabular}{lrrrrrrr} 
& \multicolumn{3}{c}{ Formulation (29) } & & \multicolumn{3}{c}{ Formulation (30) } \\
\cline { 2 - 4 } \cline { 6 - 8 } Instance & It. & PCG & CPU & & It. & PCG & CPU \\
\hline CTA-5-5-5 & $54(5)$ & 1493 & 0.51 & & $154(9)$ & 252 & 0.22 \\
CTA-10-10-5 & $94(8)$ & 4077 & 1.56 & & 41 & 1033 & 0.30 \\
CTA-15-15-10 & 141 & 87208 & 65.22 & & 66 & 3685 & 2.33 \\
CTA-20-20-20 & $200(39)$ & 189318 & 423.75 & & $200(110)$ & 13444 & 35.37 \\
CTA-25-25-10 & $162(23)$ & 114437 & 239.17 & & $109(6)$ & 29714 & 27.83 \\
CTA-25-25-25 & $200(36)$ & 105095 & 472.56 & & 200 & 5907 & 16.73 \\
CTA-30-30-10 & 147 & 35540 & 57.48 & & $187(60)$ & 45128 & 61.34 \\
CTA-30-30-30 & $200(21)$ & 387852 & 2345.41 & & $200(54)$ & 29252 & 228.41 \\
\hline
\end{tabular}

iterations was set to either 100 or 200, depending on the instance. Executions that reached this maximum limit provided a suboptimal solution. For columns "It.", the values in brackets correspond to the number of iterations performed solving normal equations by Cholesky factorizations, instead of PCG; the code automatically switches from PCG to Cholesky when a wrong solution is reported by PCG within its maximum number of iterations (set to a large value of $2 l$ ). From Tables 4-6 it is clear that the specialized interior-point algorithm is more efficient with formulation (22) than with (21), mainly due the big difference in number of PCG iterations needed by the formulations. A similar conclusion can be derived for (25) and (26), the latter being a more efficient approach. In that case, however, for (26) the code switched from PCG to Cholesky, while PCG was always enough for (25) (at the expenses of a larger 
Table 7 Results for a subset of instances comparing (22), (26) and (30)

\begin{tabular}{|c|c|c|c|c|c|c|c|c|c|}
\hline \multirow[b]{2}{*}{ Instance } & \multicolumn{3}{|c|}{ Formulation (22) } & \multicolumn{3}{|c|}{ Formulation (26) } & \multicolumn{3}{|c|}{ Formulation (30) } \\
\hline & It. & PCG & CPU & It. & PCG & $\mathrm{CPU}$ & It. & PCG & $\mathrm{CPU}$ \\
\hline CTA-40-40-20 & $200(41)$ & 14319 & 181.99 & 57 & 5256 & 49.89 & $200(61)$ & 18303 & 154.59 \\
\hline CTA-50-50-10 & $200(93)$ & 30910 & 232.18 & 54 & 5867 & 36.18 & $200(77)$ & 86882 & 315.06 \\
\hline CTA-50-50-25 & $200(65)$ & 15939 & 548.88 & $200(89)$ & 13554 & 564.70 & $200(74)$ & 23816 & 532.57 \\
\hline CTA-50-50-50 & $200(63)$ & 124991 & 6779.41 & $140(17)$ & 105453 & 4142.12 & $200(71)$ & 111273 & 4835.68 \\
\hline CTA-100-100-10 & $200(94)$ & 34339 & 1441.62 & $200(110)$ & 33176 & 1677.41 & $200(109)$ & 60610 & 1632.50 \\
\hline CTA-100-100-25 & $200(96)$ & 26844 & 5537.92 & 93(32) & 6849 & 2718.50 & $200(102)$ & 38229 & 5346.47 \\
\hline CTA-200-200-50 & \multicolumn{3}{|c|}{ Not executed } & 45 & 281 & 599.44 & \multicolumn{3}{|c|}{ Not executed } \\
\hline
\end{tabular}

number of PCG iterations). Similarly, (30) outperformed (29). This empirical evidence confirms that those formulations with fewer linking constraints are favorable for the specialized interior-point algorithm.

Table 7 reports results for the more efficient formulations (22), (26) and (30) considering the subset of larger instances. The meaning of the columns is as in previous tables. The limit of interior-point iterations was set to 200. We see that the limit was reached in many runs. In addition, the code switched from PCG to Cholesky in almost all runs. However, when this happened, the relative optimality gap between the dual and primal objective was in general $O\left(10^{-5}\right)$. The remaining - and expensiveCholesky factorization were needed to reach the required default $10^{-6}$ optimality gap. Without this interior-point iterations which solve the Cholesky factorizations of normal equations, the CPU time would have been much lower. We see that formulation (26) provides the best results, both in CPU time, but also in number of PCG and interior-point iterations. Indeed, this option was the only one that solved three instances without switching to the Cholesky factorization of normal equations. We also checked that, when this switch is done, the optimality gap reached by formulation (26) was smaller than for the other two formulations.

Finally, Table 8 shows results with the regularized interior-point algorithm, for the more efficient formulations (22), (26) and (30), and with the barrier solver of CPLEX 12.1. The meaning of the columns is as for previous tables. All the instances were considered, excluding the three largest ones for the formulations (22) and (30), since the computational time would be excessive. The value $\delta=10^{-3}$ was used for the regularization term (19). Note that for CPLEX the particular formulation considered is not relevant, since it does not exploit a particular partitioning of the constraints matrix; anyway, the results provided for CPLEX correspond to the ordering of constraints and variables of (26). From Table 8 it is clear that: (1) regularized versions are much more efficient than the non-regularized ones; (2) formulation (26) is the most efficient approach for the regularized algorithm; (3) the generic barrier of CPLEX 12.1 is the most efficient approach when the size of the problem is not very large; (4) for the tree largest instances (of millions of variables and constraints), the specialized approach outperforms the state-of-the-art interior-point solver. 
Table 8 Results comparing (22), (26) and (30), with the regularized interior-point algorithm, and the barrier solver of CPLEX 12.1

\begin{tabular}{|c|c|c|c|c|c|c|}
\hline \multirow[b]{2}{*}{ Instance } & \multicolumn{3}{|c|}{ Formulation (22) } & \multicolumn{3}{|c|}{ Formulation (30) } \\
\hline & It. & PCG & $\mathrm{CPU}$ & It. & PCG & $\mathrm{CPU}$ \\
\hline CTA-5-5-5 & 200 & 280 & 0.27 & 25 & 82 & 0.07 \\
\hline CTA-10-10-5 & 29 & 397 & 0.21 & $157(7)$ & 1894 & 0.63 \\
\hline CTA-15-15-10 & 94 & 7034 & 4.68 & 63 & 3497 & 1.95 \\
\hline CTA-20-20-20 & 36 & 2125 & 5.11 & 42 & 3140 & 4.61 \\
\hline CTA-25-25-10 & 48 & 5705 & 7.98 & 49 & 5841 & 5.60 \\
\hline CTA-25-25-25 & 58 & 2841 & 11.25 & 127 & 4233 & 12.51 \\
\hline CTA-30-30-10 & 119 & 38426 & 72.52 & 134 & 54394 & 64.11 \\
\hline CTA-30-30-30 & 100 & 14895 & 104.76 & 65 & 13753 & 55.72 \\
\hline СТА-40-40-20 & 200(39) & 19865 & 196.53 & $200(68)$ & 17893 & 139.35 \\
\hline CTA-50-50-10 & $200(58)$ & 54886 & 344.81 & $200(46)$ & 112168 & 389.36 \\
\hline CTA-50-50-25 & $200(43)$ & 21654 & 514.96 & $172(58)$ & 19365 & 369.13 \\
\hline СТА-50-50-50 & $200(56)$ & 176643 & 7762.12 & 125 & 134386 & 2509.18 \\
\hline CTA-100-100-10 & $130(26)$ & 120548 & 3382.39 & $108(7)$ & 70431 & 1337.92 \\
\hline CTA-100-100-25 & $200(79)$ & 33464 & 6607.60 & $112(8)$ & 43507 & 3225.03 \\
\hline CTA-200-200-50 & & not execut & & & not execu & \\
\hline CTA-500-500-50 & & not execut & & & not execu & \\
\hline CTA-1000-500-20 & & not execut & & & not execu & \\
\hline
\end{tabular}

\begin{tabular}{|c|c|c|c|c|c|}
\hline \multirow[b]{2}{*}{ Instance } & \multicolumn{3}{|c|}{ Formulation (26) } & \multicolumn{2}{|c|}{ barrier CPLEX 12.1} \\
\hline & It. & PCG & $\mathrm{CPU}$ & It. & $\mathrm{CPU}$ \\
\hline CTA-5-5-5 & 28 & 128 & 0.16 & 7 & 0.01 \\
\hline CTA-10-10-5 & 71 & 600 & 0.34 & 9 & 0.01 \\
\hline CTA-15-15-10 & 32 & 898 & 0.68 & 15 & 0.07 \\
\hline CTA-20-20-20 & 35 & 2011 & 4.87 & 13 & 0.73 \\
\hline CTA-25-25-10 & 39 & 2405 & 4.19 & 17 & 0.28 \\
\hline CTA-25-25-25 & 36 & 1358 & 6.37 & 9 & 1.80 \\
\hline CTA-30-30-10 & 48 & 4733 & 10.37 & 18 & 0.51 \\
\hline CTA-30-30-30 & 54 & 7865 & 52.36 & 14 & 6.85 \\
\hline CTA-40-40-20 & 46 & 3301 & 27.39 & 12 & 4.98 \\
\hline CTA-50-50-10 & 56 & 6774 & 41.36 & 28 & 2.72 \\
\hline CTA-50-50-25 & 50 & 4558 & 73.36 & 13 & 13.64 \\
\hline CTA-50-50-50 & 113 & 73002 & 1959.40 & 25 & 166.87 \\
\hline CTA-100-100-10 & 77 & 13727 & 319.79 & 30 & 24.28 \\
\hline CTA-100-100-25 & 82 & 26292 & 1604.76 & 20 & 153.92 \\
\hline CTA-200-200-50 & 42 & 282 & 514.26 & 9 & 3518.21 \\
\hline CTA-500-500-50 & 53 & 163 & 3631.98 & 9 & 55670.34 \\
\hline CTA-1000-500-20 & 51 & 160 & 2723.17 & 8 & 10050.54 \\
\hline
\end{tabular}

\section{Conclusions}

The specialized interior-point algorithm for primal block-angular problems was shown in the past to be an efficient approach for large continuous $L_{2}$-CTA instances, which result in quadratic multicommodity flow problems. However, until this work, the algorithm had not been applied to $L_{1}$-CTA. $L_{1}$-CTA has a primal block-angular structure, but it is no longer a multicommodity flow problem. However, for one of the six primal block-angular structures developed, a regularized version of the specialized interior-point algorithm has shown to be an efficient approach, outperforming the state-of-the-art interior-point solver of CPLEX 12.1 when the size of the problem is very large (millions of variables and constraints). The regularization term has shown to be instrumental in the efficiency of this approach. For larger instances, the specialized algorithm is expected to be even more efficient against CPLEX 12.1. However such massive instances can not be dealt with the current MATLAB code, and a C/C++ 
implementation of the specialized algorithm should be used. The development of this efficient implementation is one of the ongoing projects.

Acknowledgements This work has been supported by grants MTM2009-08747 of the Spanish Ministry of Science and Innovation, and SGR-2009-1122 of the Government of Catalonia.

\section{References}

Castro J (2000) A specialized interior-point algorithm for multicommodity network flows. SIAM J Opt. 10:852-877

Castro J (2005) Quadratic interior-point methods in statistical disclosure control. Comp Manag Sci. 2:107-121

Castro J (2006) Minimum-distance controlled perturbation methods for large-scale tabular data protection. Eur J Oper Res. 171:39-52

Castro J (2007a) An interior-point approach for primal block-angular problems, Comput Opt App. 36:195-219.

Castro J (2007b) A shortest paths heuristic for statistical disclosure control in positive tables. INFORMS J Comput. 19:520-533

Castro J, Cuesta J (2011) Quadratic regularizations in an interior-point method for primal block-angular problems. Math Prog. doi:10.1007/s10107-010-0341-2

Domingo-Ferrer J, Magkos E (eds.) (2010), Lecture Notes in Computer Science. Privacy in Statistical Databases (Vol. 6344). Springer, Berlin

Domingo-Ferrer J, Saigin Y (eds.) (2008), Lecture Notes in Computer Science. Privacy in Statistical Databases (Vol. 5262). Springer, Berlin

Dandekar RA, Cox LH (2002), Synthetic tabular data: An alternative to complementary cell suppression, manuscript, Energy Information Administration, US Department of Energy.

Gondzio J (1996) Multiple centrality corrections in a primal dual method for linear programming. Comput Opt App. 6:137-156

González JA, Castro J (2011) A heuristic block coordinate descent approach for controlled tabular adjustment. Comp Oper Res. doi:10.1016/j.cor.2011.02.008

Hundepool A, Domingo-Ferrer J, Franconi L, Giessing S, Lenz R, Naylor J, Schulte-Nordholt E, Seri E, de Wolf PP (2010), Handbook on statistical disclosure control ( $\mathrm{v}$ 1.2). Network of Excellence in the European Statistical System in the field of Statistical Disclosure Control. http://neon.vb.cbs.nl/casc/SDC_Handbook.pdf

Mehrotra S (1992) On the implementation of a primal-dual interior point method. SIAM J Opt. 2: 575-601

Ng E, Peyton BW (1993) Block sparse Cholesky algorithms on advanced uniprocessor computers. SIAM J Sci Comput. 14:1034-1056

Wright SJ (1996) Primal-Dual Interior-Point Methods. SIAM, Philadelphia

Zhang Y (1998) Solving large-scale linear programs by interior-point methods under the MATLAB environment. Opt Methods Soft. 10:1-31 